\title{
To Do, or Not To Do, That is the Question: Appropriateness of the Requested CT and MR Imaging Studies in the Philippine General Hospital Outpatient Department Based on the American College of Radiology Appropriateness Criteria
}

\author{
Patricia Rose L. Dairo-Mabansag, MD, MPM and Ryan Jason DL. Urgel, MD \\ Department of Radiology, Philippine General Hospital University of the Philippines Manila
}

\begin{abstract}
Objective. To determine the rate of the appropriateness of the requested CT and MRI procedures in the Outpatient Department of a tertiary hospital in the Philippines from January to June 2018 using the American College of Radiology Appropriateness Criteria (ACR-AC).
\end{abstract}

Methods. This retrospective research reviewed outpatient $C T$ and $M R$ imaging requests and cross-referenced with the corresponding ACR-AC guideline available for the written clinical diagnosis.

Results. Four hundred thirty-six (436) (56\%) of the 774 retrieved requests were CT scan studies, while the remaining 338 (44\%) are MR imaging procedures. Cross-referencing with ACR-AC, the rate of appropriateness across all patients is high at $96.6 \%$, with a $95 \%$ confidence interval of $95.0 \%$ and $97.6 \%$. The rates were not significantly different between MRI and CT ( $p$-value $=0.4502$ ). Likewise, there was no significant difference in rates of appropriateness for the body parts abdomen, cranial, chest, and spine ( $p$-value $=0.6502$ ).

Conclusion. Although the results were high, relative to the few available international studies, the importance and potential of the ACR-AC cannot be disregarded. The ACR-AC serves as a guide in selecting the appropriate imaging test given a clinical situation. This may equate to better patient management, considering all patient-related factors.

Key Words: diagnostic imaging; reviews, appropriateness; practice guideline

\section{INTRODUCTION}

Corresponding author: Patricia Rose Dairo-Mabansag, MD, MPM Department of Radiology

Philippine General Hospital

University of the Philippines Manila

Email: prdairomabansag@gmail.com
Globally, governments and private sectors have been increasing initiatives to address the prevalence of illnesses, such as cancer, cardiovascular, and infectious diseases, including tuberculosis. This remarkably contributes to the demand for and subsequent growth of diagnostic imaging and technological advancements. ${ }^{1}$

Locally, the past five years likewise saw a significant increase in the volume of diagnostic imaging examinations done in the Philippine General Hospital (PGH). In an unpublished research study done in 2016 entitled "The factors behind the increasing trend in the use of diagnostic imaging in the Philippine General Hospital from the radiologists' point of view," there was an overall increase in the volume of imaging examinations done in PGH. Notably, from 2013 to 2014, there was a note of 55\% percent increase in radiographic modality, $50 \%$ in ultrasound, $78 \%$ in 
CT scan, and 29\% in MRI. This study emphasized the implications of radiation exposure risk, as well as the economic impact, which is out-of-pocket in the majority of the Filipinos, that come with increased imaging utilization. The lack of communication between radiologists and requesting physicians was identified as one of the significant factors for the increase in diagnostic imaging use in $\mathrm{PGH} .^{2}$

This trend has been recognized long before the $21^{\text {st }}$ century, along with progressive advancements in imaging technology, and radiation safety awareness and practices. Thus, imaging appropriateness criteria guidelines were established. These are evidence-based guidelines for specific clinical scenarios, purposed to ensure optimal, cost-effective, and improved utilization of imaging studies. One of the most comprehensive of these guidelines is the American College of Radiologists' Appropriateness criteria (ACR-AC), which as of 2018, includes 179 diagnostic imaging and interventional radiology topics with 898 clinical variants, over 1,560 clinical scenarios and 223 unique topics. Its beginning traces back to the 1990s when national guidelines were needed to address the arising challenges that came with imaging advancements. A task force and panels were established, and the primary methodology of guideline creation is combination primarily of evidence from medical practice guidelines, scientific outcome, and technology assessment studies, and expert consensus, while engaging the requesting medical specialists to provide clinical perspectives. ${ }^{3}$

The ACR-AC includes guidelines for diagnostic imaging selection, radiotherapy protocols, and imageguided interventional procedures. In the diagnostic category, guidelines are sorted on a system-based classification and include breast, cardiovascular, gastrointestinal, major trauma, musculoskeletal, pediatric, thoracic, urologic, neuroradiologic, and women's imaging. For each diagnostic category, there are several topics to choose from, which may be a symptom (such as palpable breast mass) or a procedure (such as breast cancer screening). Under each topic, there are clinical variants that further characterize the clinical case. For each variant, imaging procedures are rated and categorized for its diagnostic appropriateness. The rating scale of 1 to 9 is used. These are determined and updated by consensus panels according to the available evidence-based studies as well as expert consensus in exceptional cases. The ACR-AC is usually presented in a tabulated form, which includes the diagnostic imaging type, appropriateness classification or rating, as well as the corresponding relative radiation level. An ordinal scale of 1,2,3 are categorized as "Usually not appropriate," 4, 5, 6 is under "May be appropriate," and 7, 8, 9 is "Usually appropriate."

The appropriateness categorization is also based on the included 'Summary of Literature Review' in each guideline report. "Usually not appropriate" is assigned when harm outweighs the benefits of doing the procedure, and "Usually appropriate" is when benefits outweigh the harms or risks. The category "Maybe appropriate" is when the risk-benefit ratio is equivocal. ${ }^{4}$ Relative radiation level, which is a radiation dose that estimates population total radiation risk associated with an imaging procedure, is an essential factor in choosing the appropriate imaging procedure and thus included. ${ }^{5}$ With the large number of different cases addressed by ACR-AC, these guidelines are made accessible online and as mobile applications.

With the information provided by the ACR-AC, there is added available reference for the requesting physicians as the basis for their imaging requests. For example, using the term breast evaluation, the initial search will reveal the phrases "breast cancer screening," "breast pain," and "palpable breast masses." This can be further narrowed down by specifying and choosing the narrative for palpable breast masses. Doing this will showcase or clinical variants. For each of these clinical scenarios, the radiologic procedures, along with the corresponding appropriateness rating (1-9), references for the recommendation, and the relative radiation level, will be presented in a tabulated form. Following the example of "palpable breast masses" in a case of a 40-year-old and above female, who came in for an initial evaluation (variant 1), mammography and digital breast tomosynthesis are of equal preference as initial imaging, both with an appropriateness rating of 9 (Figure 1$)^{3}$

The ACR-AC was created out of the need for and has been generally able to address the improvement of diagnostic imaging quality, reduce unnecessary imaging, and lower costs. ${ }^{3}$ Several studies have been conducted internationally, evaluating the utilization and impact of ACR-AC.In 2016,23 years since its conception, a meta-analysis was done by Sheng et al., showed low utilization and incorporation of ACR-AC in the practice of both radiologists and non-radiologists. ${ }^{6} \mathrm{In}$ another study, Levy et al., conducted a comparison of the rate of appropriateness of imaging studies, which demonstrated improvement upon introduction of the ACR criteria to general practitioners. ${ }^{7}$ The latter study shows the possible result if efforts to introduce, train, incorporate into practice, and collaborate with partner clinicians, are sustained.

In the Philippines, as local efforts increase to improve radiation safety protection practices, minimize radiation exposure risks, and maximize health funding, it is judicious to incorporate practices that can potentially address such issues.

\section{OBJECTIVE}

To determine the rate of the appropriateness of the requested $\mathrm{CT}$ and $\mathrm{MR}$ imaging procedures in the Outpatient Department of the Philippine General Hospital, from January to June 2018 using to the American College of Radiology Appropriateness Criteria

\section{METHODS}

This is a retrospective study reviewing all the CT and MR imaging requests and results done in the Department 
Variant 1: $\quad$ Palpable breast mass. Female, 40 years of age or older, initial evaluation. (See $\underline{\text { Appendices }}$ 1A-1B for additional steps in the workup of these patients.)

\begin{tabular}{|c|c|c|c|}
\hline Radiologic Procedure & Rating & Comments & RRL* \\
\hline Mammography diagnostic & 9 & See references [13-15]. & (2) \\
\hline Digital breast tomosynthesis diagnostic & 9 & See references $[16-18,20,85]$. & (2) \\
\hline US breast & 4 & $\begin{array}{l}\text { If she had recent mammogram (ie, past } 6 \\
\text { months), US may be appropriate. } \\
\end{array}$ & $\mathrm{O}$ \\
\hline MRI breast without and with IV contrast & 2 & See references $[4,49]$. & O \\
\hline MRI breast without IV contrast & 1 & & $\mathrm{O}$ \\
\hline FDG-PEM & 1 & & (4) (3) (3) \\
\hline Sestamibi MBI & 1 & & (2) \\
\hline Image-guided core biopsy breast & 1 & & Varies \\
\hline Image-guided fine-needle aspiration breast & 1 & & Varies \\
\hline \multicolumn{3}{|c|}{ Rating Scale: 1,2,3 Usually not appropriate; 4,5,6 May be appropriate; 7,8,9 Usually appropriate } & elative \\
\hline
\end{tabular}

Figure 1. Example of ACR-AC guideline with "palpable breast masses" narrative and case variant 1 as detailed. ${ }^{3}$

of Radiology, Philippine General Hospital from January to June 2018.

A digital copy of the results was accessed from the Local Area Network of the Department of Radiology of PGH. Each record was reviewed and sorted according to the inclusion and exclusion criteria listed below.

\section{Inclusion criteria}

To identify outpatient studies, Out Patient Department (OPD) identifiers including "OPD, outpay, 1AO4, 2B05, 1BO4, 1CO6, 2CO2, 2B09 and 2AO5" and similar identifiers were used as the key or search words. These were inputted in the search bar of the results in the covered time period. CT and MRI records with available results either provisionally or officially interpreted, were included.

\section{Exclusion criteria}

Incompletely and improperly filled up imaging requests, such as those lacking the clinical indication, were excluded.

Among those included in the study, most of the patient identifiers were removed. Only the age and gender were gathered among the patient demographics. Included requests were codified as "ACR-AC1, ACR-AC2, ACR-AC3..." and so on. These data were stored in a personal research folder of the author in the local network of the department, which was protected with a password, accessible only to the primary investigator. Gathered data were stored until the study implementation and final paper writing, approximately 6 months from the start of the study.

For each imaging request, the type of study (CT or MRI), body part examined (brain, head and neck, chest, abdomen, musculoskeletal or spine), and clinical indication, were recorded. The guideline for appropriateness criteria, accessed online from the ACR website, was applied by the primary investigator, with the adviser clinching questionable cases as needed. Depending on the written clinical indication, it will be used against the ACR-AC and check for the appropriateness of the specific test on the given case.

Patient demographics and imaging procedure information were summarized using the mean and standard deviation for numerical variables, and frequency and percentage for categorical variables. The rate of appropriateness was computed as a percentage, and a $95 \%$ confidence interval based on the normal distribution is constructed. The chisquare test was used to compare the appropriateness of the requested imaging study and the clinical indication.

\section{Ethical Considerations}

The protocol for this study was submitted to the University of the Philippines Manila Research Ethics Board (UPMREB) Panel for ethics review and approval. The implementation of the study was done only upon the approval of the review board. This study did not involve any human subjects. Only the medical records and imaging reports were reviewed. All information about the subjects were anonymized and kept confidential. A waiver of informed consent was requested from the UPMREB panel since anonymized medical records and reports were used. All funding was from the principal investigator. There was no conflict of interest. There were no foreseeable direct risks nor benefits. In cases of breach of privacy, the matter will be forwarded to the PGH Data Privacy Officer.

\section{RESULTS}

There were 774 records gathered from January to June 2018. The records were almost evenly distributed between 
Table 1. Demographic Characteristics and Imaging information

\begin{tabular}{lr}
\multicolumn{1}{c}{ Characteristic } & Statistic \\
Type of Imaging, $\mathrm{n}(\%)$ & \\
CT & $436(56.33)$ \\
MRI & $338(43.67)$ \\
\hline Sex, $\mathrm{n}(\%)$ & \\
Female & $390(50.39)$ \\
Male & $384(49.61)$ \\
Age (Mean \pm SD) & $43.2 \pm 20.66$ \\
\hline Body Part Examined, n(\%) \\
Abdomen \\
Chest & $220(28.42)$ \\
Cranial & $138(17.83)$ \\
Head and Neck (H\&N) & $197(25.45)$ \\
Musculoskeletal (MSK) & $76(9.82)$ \\
Spine & $60(7.75)$ \\
\hline
\end{tabular}

males and females, with 43 as the average age of the patients. Fifty-six percent of the patients underwent a CT scan procedure, while the remaining had MRI. The body part most commonly imaged was the abdomen (28\%), followed by brain (25\%) and chest (18\%). (Table 1$)$.

Across all imaging studies, the rate of appropriateness is $96.6 \%$, with a $95 \%$ confidence interval. The rates were not significantly different between CT and MR imaging ( $\mathrm{p}$-value $=0.4502)$. Likewise, there was no significant difference in the rates of appropriateness for the imaging of the abdomen, cranial, chest and spine ( $p$-value $=0.6502)$. (Figure 2) (Table 2)

\section{DISCUSSION}

Cross-referencing with the Appropriateness Criteria of the American College of Radiology, the requested imaging (CT and MR) studies in the outpatient services of PGH yielded a high overall rate of appropriateness at 96.6\%, with no significant difference between CT and MRI procedures. While there is not much available data on the global utilization of imaging appropriateness criteria, the yielded rate of $96.6 \%$ is much higher than the $74 \%$ over-all rate in a similar study done by Lehnert and Bree involving 459 examinations using the ACR guidelines. ${ }^{8}$ In their study, they identified that the utilization of a national Radiology
Benefits Management (RBM) program, the decision system adopted by the Medicare system in the United States at that time, contributed to the relatively higher rate of inappropriate studies. They also noted that most of their outpatient requests were seen by the radiologists during interpretation and result production, long after the procedure was done, and thus requesting physician-radiologist interaction is put to a minimum.

In the PGH setting, appropriateness criteria for imaging studies, including the ACR guidelines, is not utilized and applied. This is reflective of the international data that shows low utilization of the ACR guidelines, which was credited mostly to the lack of awareness of such guidelines by both clinicians and radiologists, as well as the clinicians' preference to inquire from their radiologists. ${ }^{9}$ This is seen similarly in the set-up in the PGH. Most of the time, doctors from the different clinical services discuss with the radiologists on what imaging study is best given the case of their patients. This, however, is not a common practice in the outpatient services, as the patients usually just go to the radiology department with their imaging requests. As the situation may differ, institutional and departmental guidelines have been in place to screen and approve the requests from the outpatient department, prior to approval, to ensure that the

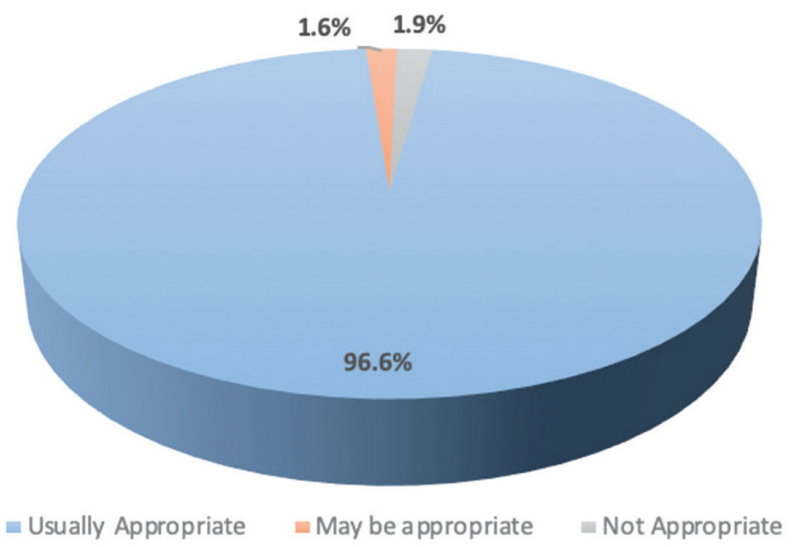

Note: 20 (2.6\%) of the images were non-classifiable.

Figure 2. Overall distribution of "Usually appropriate, May be appropriate and Usually not appropriate" tests.

Table 2. Examination-specific appropriateness rates

\begin{tabular}{|c|c|c|c|c|}
\hline \multirow{2}{*}{ Category } & \multicolumn{4}{|c|}{ ACR Classification } \\
\hline & Usually Appropriate & May be appropriate & Not Appropriate & Not Classifiable \\
\hline \multicolumn{5}{|l|}{ Type of Imaging, n(\%) } \\
\hline $\mathrm{CT}(\mathrm{N}=436)$ & $415(95.2)$ & $8(1.8)$ & $6(1.4)$ & $7(1.6)$ \\
\hline $\mathrm{MRI}(\mathrm{N}=338)$ & $313(92.6)$ & $4(1.2)$ & $8(2.4)$ & $13(3.8)$ \\
\hline \multicolumn{5}{|l|}{ Body Part Examined, n(\%) } \\
\hline Abdomen $(N=220)$ & 207 (94.1) & $3(1.4)$ & $6(2.7)$ & $4(1.8)$ \\
\hline Chest $(\mathrm{N}=138)$ & $133(96.4)$ & $2(1.4)$ & $1(0.7)$ & $2(1.4)$ \\
\hline Cranial $(\mathrm{N}=197)$ & $182(92.4)$ & $5(2.5)$ & $3(1.5)$ & $7(3.6)$ \\
\hline$H \& N(N=76)$ & 73 (96.1) & & $1(1.3)$ & $2(2.6)$ \\
\hline $\operatorname{MSK}(N=60)$ & $57(95.0)$ & $1(1.7)$ & & $2(3.3)$ \\
\hline Spine $(N=83)$ & 76 (91.6) & $1(1.2)$ & $3(3.6)$ & $3(3.6)$ \\
\hline
\end{tabular}


right test will be done given the clinical history. This may have contributed to the better appropriateness rate in our outpatient services. The knowledge of most physicians on what appropriate imaging procedure to be done likewise adds to the better adherence to the appropriateness criteria.

Twenty out of the 774 outpatient requests (about 2.6\%), were labeled "Not classifiable." These are mostly because the written clinical diagnosis is not covered by the ACR guidelines. Some congenital diseases and syndromes (Hunter and Proteus syndromes) are among those not included in any of the clinical vignettes in the ACR Appropriateness criteria. Five of these even lack clinical information.

Fourteen of the requests (1.9\%) were marked as "Usually not appropriate." These are procedures that were done but have demonstrated more harm than good. For MRI procedures, these include imaging the abdomen to look for anal pain and hernia, and the spine for osteoporosis, cognitive impairment, and traumatic post-operative intervention follow-up. For CT procedures, requests for imaging the following were deemed as inappropriate: brain CT for chronic headache, chest CT for a routine check-up, as well as abdominal CT for increased kidney size, benign prostatic hypertrophy, and constipation. Most of these are different from those identified as inappropriate examinations in the study by Lehnert and Bree, except for the brain CT for chronic headache. ${ }^{8}$ While some of these clinical scenarios can be cross-referenced easily with the ACR-AC guidelines, which can be accessed online, a definite ACRAC classification is not easily accessed, consuming about 2-5 minutes of thorough searching, compared to the average of 30-60 seconds for the other clinical keywords. While ACR is the most comprehensive appropriateness imaging guideline, certain cases are not yet incorporated, such as congenital diseases and syndromes, which may need tailored diagnostic examinations. Unfamiliarity with the ACR-AC guidelines as well as varied medical keywords used may have contributed to the increased time in cross-referencing, particularly of the 'inappropriate' requests.

\section{CONCLUSION AND RECOMMENDATIONS}

The American College of Radiology Appropriateness Criteria may be a helpful and comprehensive tool in delivering quality and cost-effective health services. While it is not implemented in the Philippine General Hospital, there is a remarkably high rate of the appropriateness of the requested CT and MRI examinations in the outpatient department. Instituted departmental practices and policies may have contributed to such outcomes, which include screening and approval of the requests by the radiologists, and constant discussions with the doctors from the different clinical services in the form of multi-disciplinary conferences or even from simple visits to the radiology department.

Although the results showed highly appropriate requesting practices of the doctors of the Philippine General
Hospital, the importance and potential of the ACR-AC cannot be disregarded. As such, the unfamiliarity of the doctors with such guidelines needs to be addressed, should this be instituted in the said hospital soon.

Even with the potential of using the ACR-AC guidelines in the Philippine General Hospital, it will be helpful to know the necessary knowledge and current practices of the clinicians and radiologists regarding the said guidelines. Information gathered with such a study can be used in the future to determine if the guidelines will have an impact on the management of patients.

\section{Statement of Authorship}

Both authors participated in data collection and analysis, and approved the final version submitted.

\section{Author Disclosure}

Both authors declared no conflicts of interest.

\section{Funding Source}

All expenses in the conduct of this study were shouldered by the authors.

\section{REFERENCES}

1. Hub M. Global Market Study on Diagnostic Imaging Devices: AsiaPacific to Witness Highest Growth by 2020 [Internet]. 2015 [cited May 2018] Available from https://www.persistencemarketresearch. $\mathrm{com} /$ market-research/diagnostic-imaging-devices-market.asp.

2. Masangya DC. The factors behind the increasing trend in the use of diagnostic imaging in the Philippine General Hospital from the radiologists' point of view. 2016. (unpublished). Available at: http://philippinecollegeofradiology.org.ph/wppcr/wp-content/ uploads/2017-PCR-Research-Journal3.pdf

3. ACR Appropriateness Criteria [Internet]. 2018 [cited May 2018]. Available from https://www.acr.org/Clinical-Resources/ACRAppropriateness-Criteria.

4. ACR Appropriateness Criteria ${ }^{\circledR}$ Rating Round Information [Internet]. 2018 [cited May 2018]. Available from https://www.acr.org/-/ media/ACR/Files/Appropriateness-Criteria/RatingRoundInfo.pdf.

5. ACR Appropriateness Criteria ${ }^{\circledR}$ Radiation Dose Assessment Introduction [Internet]. 2018 [cited May 2018]. Available from https://www.acr.org/-/media/ACR/Files/Appropriateness-Criteria/ RadiationDoseAssessmentIntro.

6. Sheng AY, Castro A, Lewiss RE. Awareness, Utilization, and Education of the ACR Appropriateness Criteria: A Review and Future Directions. J Am Coll Radiol. 2016 Feb;13(2):131-6. doi: 10.1016/ j.jacr.2015.08.026. Epub 2015 Oct 21.

7. Levy G, Blachar E, Goldstein L, et al. Nonradiologist Utilization of American College of Radiology Appropriateness Criteria in a Preauthorization Center for MRI Requests: Applicability and Effects. American Journal of Roentgenology. 2006 Oct;187(4):855-8. doi: 10.2214/AJR.05.1055

8. Lehnert BE, Bree RL. Analysis of Appropriateness of Outpatient CT and MRI Referred From Primary Care Clinics at an Academic Medical Center: How Critical Is the Need for Improved Decision Support? J Am Coll Radiol. 2010 Mar;7(3):192-7. doi: 10.1016/ j.jacr.2009.11.010.

9. Bautista AB, Burgos A, Nickel BJ, Yoon JJ, Tilara AA, Amorosa JK. Do Clinicians Use the American College of Radiology Appropriateness Criteria in the Management of Their Patients? AJR Am J Roentgenol. 2009 Jun;192(6):1581-5. doi: 10.2214/AJR. 08.1622 . 